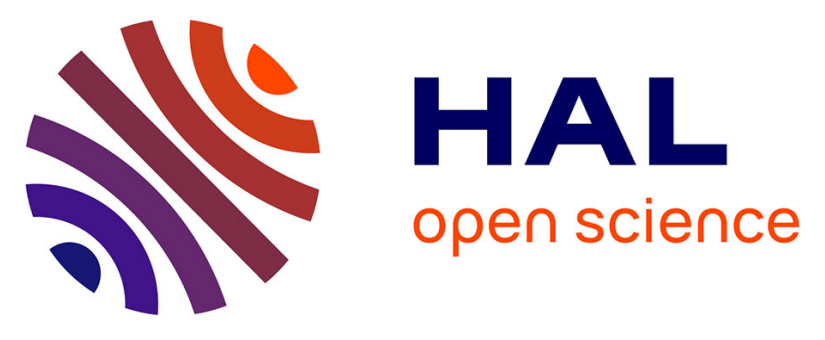

\title{
Neonatal Maternal Deprivation Promotes Nippostrongylus Brasiliensis Infection in Adult Rats
}

Frédéric Barreau, Jacques Ducos de Lahitte, Laurent Ferrier, Rafael Garcia Villar, Lionel Bueno, Jean Fioramonti

\section{To cite this version:}

Frédéric Barreau, Jacques Ducos de Lahitte, Laurent Ferrier, Rafael Garcia Villar, Lionel Bueno, et al.. Neonatal Maternal Deprivation Promotes Nippostrongylus Brasiliensis Infection in Adult Rats. Digestive Disease Week / 106th Annual meeting of the AGA, May 2005, Chicago, Illinois, United States. 2005, Gastroenterology 128 (4 suppl 2). hal-01604779

\section{HAL Id: hal-01604779 \\ https://hal.science/hal-01604779}

Submitted on 4 Jun 2020

HAL is a multi-disciplinary open access archive for the deposit and dissemination of scientific research documents, whether they are published or not. The documents may come from teaching and research institutions in France or abroad, or from public or private research centers.
L'archive ouverte pluridisciplinaire HAL, est destinée au dépôt et à la diffusion de documents scientifiques de niveau recherche, publiés ou non, émanant des établissements d'enseignement et de recherche français ou étrangers, des laboratoires publics ou privés.

\section{다(1)(2)}

Distributed under a Creative Commons Attribution - ShareAlikel 4.0 International 


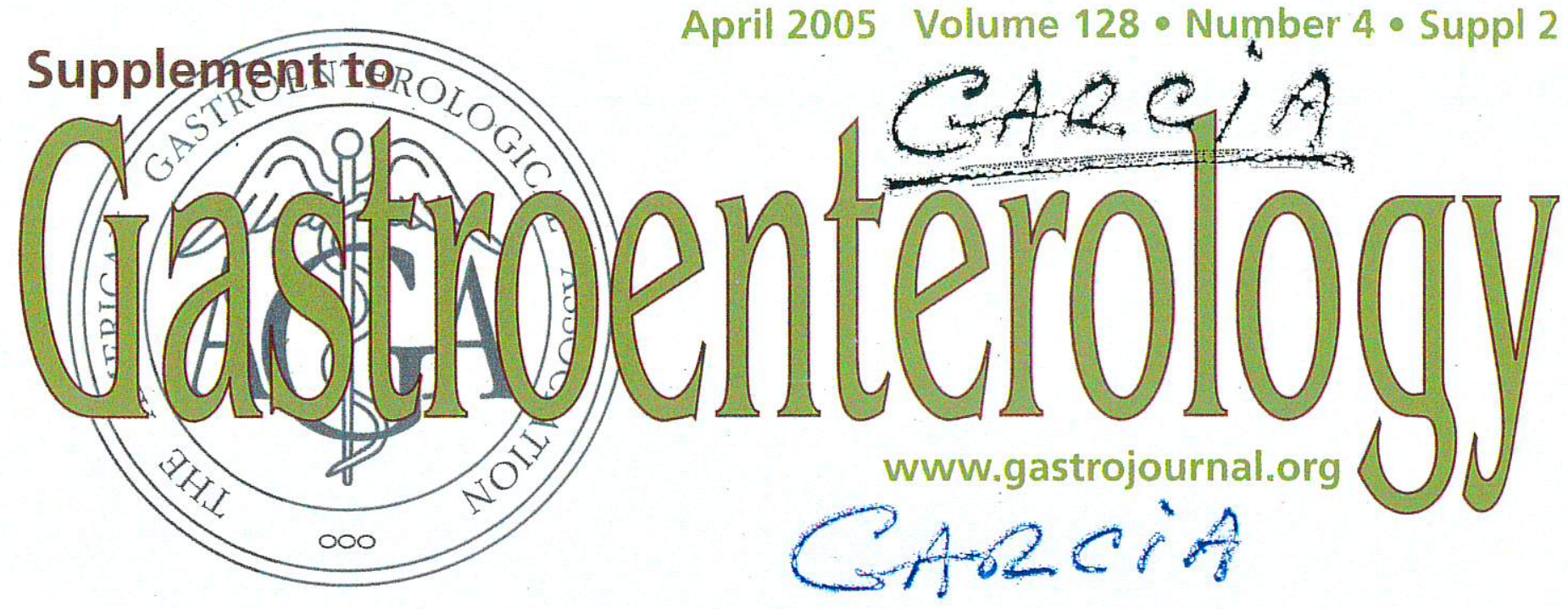

April 2005 Volume $128 \cdot$ Number $4 \cdot$ Suppl 2

Digiestive Disease $\mathbb{W}^{-e e k}{ }^{\oplus}$

and the

106th Anmual Meeting of the American

Gastroenterological Association May 14-19, 2005, Chicago, IL

Program of the Annual Meeting of the American Gastroenterological Association, the American Association for the Study of Liver Diseases, the Gastroenterology Research Group, the Society for Surgery of the Alimentary Tract, and the American Society for Gastrointestinal Endoscopy

Albstracts of Papers Accepted by the American Gastroenterological Association

Abstracts of Papers Accepted by the American Association for the Study of Liver Diseases

Abstracts of Papers Accepted by the Society for Surgery of the Alimentary Tract 
provide valuable insight for design of selective mucosal modulators of IFN- $\gamma$ expression in the gut.

$763 \mathrm{~A}$

Negative Regulation of TNFR2 Cascades by TNFR1 Contributes to the Suppression of Colitis

of Colitis
Atsuhiro Ogawa*, Ken Sugimoto, Atsushi Mizoguchi, Emiko Mizoguchi, Atul K. Bhan

BACKGROUND\&AIMS: TNFa and consequently TNFa receptor typel (TNFR1) and type2 (TNFR2) play a critical role in the pathogenesis of IBD. The mechanism by which TNFa (TNFR2) play a critical role in the pathogenesis of IBD. The mechanism by which TNFa
operates colitis is known to be very complex and multi-factorial due to the presence of two receptors. However, the functional role of interaction between TNFRI and TNFR2 in colitis has not been fully elucidated yet. METHODS: To study the roles of TNFRs in a Th2 mediated colitis, TNFR1 $\times$ TCRa double knockout (DKO) $(n=18)$, TNFR2 $\times$ TCRa DKO $(n=29)$, and TNFR1 $\times$ TNFR2 $\times$ TCRa TKO triple knockout (TKO) $(n=20)$ mice were generated. The presence of colitis was evaluated at 6 months of age by gross and histological examinations. The changes of phenotypes and apoptosis-associated signaling cascades in the colonic lamina propria (LP) cells were examined by real-time PCR and FACS analyses. the colonic lamina propria (LP) cells were examined by real-time PCR and FACS analyses,
RESULTS: As previously demonstrated (Gastroenterology 2002,122:134), mild form of colitis with low frequency developed in TNFR2 x TCRa DKO mice compared to TCRa KO mice. In contrast, TNFRI x TCRa DKO mice compared to TCRa KO mice developed much more severe colitis that is associated with a significant increase in colonic LP CD4 + T cells Interestingly, TNFR2 expressions were further upregulated on the colonic LP CD4+ T cells of TNFR1 x TCRa DKO mice compared to TCRa KO mice. In addition, the absence of TNFR1 led to the downregulation of apoptosis-associated molecules including $\mathrm{BH} 3$ interacting domain death agonist (Bid), Bcl-associated death promoter (Bad), and apoptosis-associated speck-like protein containing a CARD (ASC) in the colonic LP CD4+ T cells. To test whether the exacerbated colitis observed in TNFR1 x TCRa DKO mice results from the enhanced activation of TNFR2 cascades, TCRa triple knockout mice deficient in both TNFR1 and TNFR2 were generated. Of note, like TNFR2 x TCRa DKO mice, significantly less severe colitis with low frequency was observed in the TNFR1 $x$ TNFR2 $\times$ TCRa TKO mice compared to TCRa KO and TNFRI $x$ TCRa DKO mice.

CONCLUSION: These studies provide an evidence to address TNFRI-associated signaling cascades as a negative regulator of TNFR2 pathway in colonic LP CD4+ T cells. TNFR1 contributes to the suppression of a Th2-mediated colitis developing in TCRa KO mice.

764

The Anti-Tumour-Necrosis Factor-Alpha Agents Infliximab and Onercept Induce P38 Mitogen Activated Protein Kinase-Dependent Apoptosis in Monocytes Via Reverse TNF Signaling and Autocrine Transforming Growth Factor-Beta 1 Philip C. Rosenstiel, Georg H. Waetzig, Alexander Arlt, Andreas Till, Heiner Schaefer, Dirk Seegert, Stefan Schreiber

Background \& Aims: The pro-inflammatory cytokine tumor necrosis factor-alpha (TNFalpha) plays a central role in inflammatory disorders. There is growing evidence that the efficacy of anti-TNF-alpha therapies in steroid-refractory Crohn's disease (CD) may critically depend on the binding of the transmembrane precursor of TNF-alpha (mTNF-alpha), thus eliciting complex intracellular signaling events (e.g. p38 mitogen-activated protein kinase (MAPK) activation and apoptosis), a process described as 'reverse signaling'. The aim of the (MAPK) activation and apoptosis), a process described as 'reverse signaling'. The aim of the present study was to elucidate the differential signaling mechanisms of the two unrelated TNF-
binding agents infliximab (chimeric anti-TNF-alpha antibody) and onercept (soluble TNFR1). Methods: Signaling pathways induced by ligation of mTNF-alpha were investigated using phospho-specific Western blots and reporter gene assays. Apoptosis was assessed using MTT assays, caspase activity measurements, Annexin V FACS. and stable transfection of dominantnegative forms of key apoptotic molecules (dnFADD/dnFLICE). Transforming growth factor (TGF)-beta levels and their autocrine action on TGF-receptors were assessed by ELISA, blocking antibodies and siRNA approaches.

Results: Infliximab and onercept exert immunoregulatory functions by induction of deathResults: Infliximab and onercept exert immunoregulatory functions by induction of deathline THP-1 through reverse signaling via mTNF-alpha. Molecular mechanisms of mTNFalpha-induced apoptosis involve release of cytochrome $\mathrm{c}$ and activation of caspase 9 and 3 . Expression of dnFLICE, but not dnFADD protein could attenuate cell death. Interference with MAPK pathways indicates that $\mathrm{p} 38$ alpha and ERK1/2 have contrasting functions in the apoptotic cascade triggered by sTNFRI via a TGF-beta autocrine loop. While p38alpha apoptotic cascade triggered by sTNFRI via a TGF-beta autocrine loop. While p38alpha
activity is essential for sTNFR1-induced apoptosis, ERK1/2 has a protective function. Interestingly, these effects seem to depend on an autocrine loop of TGF-beta, as inhibition of TGFbeta signaling reduce both MAPK activation and apoptosis.

Conclusions: This mechanism unveils a novel aspect of the molecular function of TNFalpha-blocking agents in CD therapy, but also suggests a physiological role of sTNFR1 in immune regulation beyond a mere neutralization of soluble TNF-alpha. Molecular dissection of TGF-beta-dependent apoptotic and non-apoptotic signaling induced by mTNF-alpha ligation may have important implications for the design of future therapeutic strategies in IBD.

765

Neonatal Maternal Deprivation Promotes Nippostrongylus Brasiliensis Infection in Adult Rats.

Frèdérick Barreau, Jacques Ducos de Lahitte, Laurent Ferrier, Rafael Garcia-Villar, Lionel Bueno, Jean Fioramonti

Background: Neonatal stress is known to alter immune responses (macrophages, mast cells and lymphocyte activity, and Th1 and Th2 cytokines expression) in adults, and parasite infection is modulated by the immune status of the host. The present study aimed to establish whether immune alterations induced by maternal deprivation affects the time course of Nippostrongylus brasiliensis infection and the associated intestinal alterations in adult rats.
Methods: Male Wistar rat pups were separated from their mother ( 3 hours/day) during postnatal days 2 to 14 (deprived rats), or left undisturbed (control). At 12 weeks of age, $\mathrm{N}$. brasiliensis infection was performed by subcutaneous administration of $3000 \mathrm{~L} 3$ infective larvae. On days 7 and 12 after this primary infection, the number of adult worms in the jejunum, the fecal egg output, paracellular jejunal permeability (FITC-dextran flux in Ussing chamber) and jejunal myeloperoxidase (MPO) activity were measured. A secondary infection ( $3000 \mathrm{~L} 3$ larvae) was performed 21 days after the first one. On days 7 and 12 after this second infection, the number of jejunal adult worms and fecal eggs were counted. Results second infection, the number of jej
were expressed as the mean \pm SEM

Results: On the 7th day after a primary infection, maternal deprivation increased the number of jejunal adult worms $(805 \pm 75$ vs $536 \pm 54 ; \mathrm{P}<0.05)$ and the number of eggs in the feces $(18060 \pm 2669$ vs $10560 \pm 1426 ; \mathrm{P}<0.05)$. On the 12 th day, adult worms were only observed in adult deprived rats. In absence of infection and in comparison with controls, maternal deprivation increased jejunal MPO activity $(229.5 \pm 43.5$ vs $92.4 \pm 11.1 \mathrm{U} / \mathrm{g}$ tissue $)$ and paracellular permeability $(11.6 \pm 1.1 \mathrm{v5} 7.3 \pm 0.4 \mathrm{nmo} / \mathrm{h} / \mathrm{cm}$ ) In control rats, N. brasiliensis infection (day 7) increased MPO activity $(162.9 \pm 43.9$ vs $92.4 \pm 11.1 \mathrm{U} / \mathrm{g}, \mathrm{P}<0.05)$ sis infection (day 7$)$ increased MPO activity $(162.9 \pm 43.9$ vs $92.4 \pm 11.1 \mathrm{U} / \mathrm{g}, \mathrm{P}<0.05)$
and paracellular permeability $(11.2 \pm 0.6 \mathrm{vs} 7.3 \pm 0.4 \mathrm{nmol} / \mathrm{h} / \mathrm{cm}, \mathrm{P}<0.05)$. Infection in deprived rats enhanced the increase of MPO activity $(385.6 \pm 44.8$ vs $229.5 \pm 43.5 \mathrm{U} / \mathrm{g}$. $\mathrm{P}<0.05)$ but not paracellular permeability $(12.4 \pm 0.9 \mathrm{vs} 11.6 \pm 1.1 \mathrm{nmol} / \mathrm{h} / \mathrm{cm} \quad ; \mathrm{P}>0.05)$. After the second infection, no adult worm and fecal egg were detected in both control and deprived rats.

Conclusions: Neonatal maternal deprivation favors a primary infection by $\mathrm{N}$. brasiliensis in Conclusions: Neonatal maternal deprivation lavors a primary infection by N. brasiliensis in
adult rats and enhances the jejunal inflammation induced by the parasite. However, maternal deprivation does not induce severe breakdown of immunity to N. brasiliensis, since the parasite does not develop after a secondary infection.

766

Hibernation Confers Resistance To Organ Damage Following Intestinal IschemiaReperfusion

Courtney C. Fleck, Susanne L. Lindell, Martin J. Mangino, Hannah V. Carey
Reperfusion

Mammalian hibernation is accompanied by a suite of physiologic events that are life-threatening for non-hibernating species such as humans. These include extended fasting (up to 6 months) and major changes in intestinal blood flow as animals repeatedly cycle into and out of the torpid state. We hypothesized that endogenous defense mechanisms that minimize injury to intestinal tissues during the hibernation season may also increase resistance to imposed intestinal ischemia-reperfusion (I/R) injury. We used a model of superior mesenteric artery (SMA) occlusion $(20 \mathrm{~min})$ followed by reperfusion $(60 \mathrm{~min})$ in rats, summer ground squirrels, and hibernating squirrels that had fully aroused between bouts of torpor (i.e., all animals had body temperatures of $37-38^{\circ} \mathrm{C}$ ). Sham animals from each group were anesthetized and the SMA was isolated, but not occluded. Intestinal I/R induced severe damage to the ileum with massive epithelial shedding and mucosal damage in rats and summer squirrels, but not hibernators. Histological damage scores were 3.7 -fold higher in $1 / R$ vs sham rats and 3.4-fold higher in $\mathrm{I} / \mathrm{R}$ vs. sham summer squirrels ( $\mathrm{p}<0.01$ for both). In contrast, there was no difference in damage scores for $I / R$ vs. sham hibernators $(p=0.16)$. We assessed neutrophil invasion into remote organs by mucosal myeloperoxidase activity (MPO) Compared with sham animals, MPO activity after I/R increased significantly in liver (6-fold) and pared with sham animals, MPO activity alter I/R increased significantly in liver (6-fold) and
lung (3-fold) in rats but not in the hibernators. The results support the hypothesis that the hibernation phenotype provides enhanced protection against intestinal I/R injury. Identification of mechanisms that underlie this effect may provide new targets for therapeutic treatment or pretreatment to minimize intestinal $1 / R$ injury in humans. Supported by US Army grant DAADI90110455.

767

Intraluminal Oxygen Prevents Mucosal Ischemic Damage in a New Model of Complete Local Small Bowel Ischemia in Pigs Peter BF Mensink, Claudius Kruse, Jeroen J Kolkman, Bernward Reszel, Uvo Hölscher, Arjen de Weerd, Gisbert Knichwitz

Introduction

Microvascular ischemia of the gastrointestinal tract is an important, and difficult to treat disorder. It is an important mechanism underlying e.g. radiation proctitis, Crohn's disease and anastomotic leakage after esophageal and colorectal resections. In the latter, it is the main contributor to anastomic leakage, morbidity and mortality. We explored a new approach by applying intraluminal oxygen in an animal model with complete local GI-ischemia. Methods

In large pigs, a $70 \mathrm{~cm}$ small bowel loop was isolated, vessels were ligated and 2 tubes placed intraluminally at both ends; over these tubes tight ligatures were placed, thereby interrupting the collateral intramural vessels, creating complete small bowel ischemia. Complete hemodynamic monitoring, as well as blood gas sampling, was performed. Oxygen $(5 \mathrm{ml} / \mathrm{min})$ was administered intraluminally in the middle part of the ischemic loop, via a multi channel catheter connected to a prototype applicator. Mucosal blood flow and oxygen saturation were measured. Complete ischemia was maintained for $2 \mathrm{hr}$. Afterwards, transmural tissue samples were taken for histopathology from the ischemic loop (in the middle part, 10, 20 and $30 \mathrm{~cm}$ proximal and distal). Mucosal damage was graded according to the Chiu classification (grade 0 to 4)

Results

Six pigs received no oxygen (sham) and 6 pigs received oxygen (treatment). Hemodynamic parameters remained stable and did not differ between both groups; no evidence of systemic inflammatory response was found. In both groups no mucosal blood flow was detectable. The sham-group showed severe ischemia, the treated group showed macroscopically almost 